PROCEEDINGS OF THE

AMERICAN MATHEMATICAL SOCIETY

Volume 130, Number 12, Pages 3605-3608

S 0002-9939(02)06608-X

Article electronically published on May 8, 2002

\title{
ON A PROBLEM OF J. P. WILLIAMS
}

\author{
EDWARD KISSIN AND VICTOR S. SHULMAN
}

(Communicated by David R. Larson)

\begin{abstract}
Let $B(H)$ be the algebra of all bounded operators on a Hilbert space $H$. Let $g$ be a continuous function on the closed disk $D$ and let

$$
\|g(A) X-X g(A)\| \leq C\|A X-X A\|,
$$

for some $C>0$, for all $X \in B(H)$ and all $A \in B(H)$ with $\|A\| \leq 1$. Then $g$ is differentiable on $D$. The paper shows that the function $g$ may have a discontinuous derivative.
\end{abstract}

\section{INTRODUCTION}

Let $B(H)$ be the algebra of all bounded operators on a Hilbert space $H$ and $\mathbf{B}_{1}$ be the unit ball of $B(H)$. For $A, B \in B(H)$, we denote by $[A, B]$ their commutator $A B-B A$. Let $D=\{z \in \mathbb{C}:|z| \leq 1\}$ be the closed unit disk. In his paper [7] Williams raised the following problem. If $g$ is a continuous complex-valued function on $D$, possessing the property

$$
\|[g(A), X]\| \leq C\|[A, X]\|,
$$

for some $C>0$, for any $X \in B(H)$ and any normal operator $A$ in $\mathbf{B}_{1}$, must $g$ always be continuously differentiable on $D$ ?

It should be noted that Johnson and Williams proved earlier [2, Theorem 4.1] that $g$ must be differentiable on $D$ and therefore analytic in the interior $D^{\circ}$ of $D$, and its derivative must be bounded on $D$.

We will show that the answer to Williams's problem is negative. Moreover, we will show that the function on $D$ may have a discontinuous derivative even if it satisfies (1) for all (not necessarily normal) contractions $A$.

The authors are grateful to the referee for his useful suggestions.

\section{Fully Operator Lipschitz functions}

We denote by $\mathcal{A}(D)$ the disk algebra: the algebra of all continuous complexvalued functions on $D$ which are analytic on $D^{\circ}$. The algebra $\mathcal{A}(D)$ is a closed subalgebra of the algebra $C(D)$ of all continuous complex-valued functions on $D$ with the norm $\|g\|=\sup _{z \in D}|g(z)|$. The subalgebra $P(D)$ of all polynomials on $D$ is dense in $\mathcal{A}(D)$ (see, for example, [4 §3.2.13]).

Received by the editors March 19, 2001 and, in revised form, July 6, 2001.

2000 Mathematics Subject Classification. Primary 47A56.

(C)2002 American Mathematical Society 
By von Neumann's theorem (see [6, Proposition I.8.3]), $\|p(A)\| \leq\|p\|$ for any polynomial $p$ and any $A \in \mathbf{B}_{1}$. Therefore functions from $\mathcal{A}(D)$ act on $\mathbf{B}_{1}$ and

$$
\|g(A)\| \leq\|g\|, \quad \text { for any } g \in \mathcal{A}(D) \text { and } A \in \mathbf{B}_{1} .
$$

We call a function $g \in \mathcal{A}(D)$ Fully Operator Lipschitzian if there is $C>0$ such that

$$
\|g(A)-g(B)\| \leq C\|A-B\|, \quad \text { for } A, B \in \mathbf{B}_{1} .
$$

The class of Fully Operator Lipschitz functions is contained in the wider class of Operator Lipschitz functions on $D$ which consists of all continuous functions on $D$ satisfying inequality (3) for all normal operators in $\mathbf{B}_{1}$ (see [3]). The function $g(z)=\bar{z}$, for example, is Operator Lipschitzian on $D$, since $\left\|A^{*}-B^{*}\right\|=\|A-B\|$, for all normal $A, B \in \mathbf{B}_{1}$. However, it is not Fully Operator Lipschitzian. Both classes of functions are important for applications in mathematical physics and have attracted much attention (see, for example, Bibliography in [1]).

Proposition 1. A function $g \in \mathcal{A}(D)$ is Fully Operator Lipschitzian if and only if there is $C>0$ such that

$$
\|[g(A), X]\| \leq C\|[A, X]\|, \quad \text { for } A \in \mathbf{B}_{1} \text { and } X \in B(H) .
$$

Proof. If $A, B \in \mathbf{B}_{1}$, the operator $L=\left(\begin{array}{ll}A & 0 \\ 0 & B\end{array}\right)$ on $H \oplus H$ belongs to the unit ball of $B(H \oplus H)$. Let $X=\left(\begin{array}{ll}0 & 1 \\ 0 & 0\end{array}\right)$. Clearly, (44) holds for operators on $H \oplus H$. Hence $\|[g(L), X]\| \leq C\|[L, X]\|$ which implies (3).

Conversely, let $\|A\|<1$. For $X \in B(H)$, the operators $A(t)=e^{t X} A e^{-t X}$ belong to $\mathbf{B}_{1}$ for sufficiently small $t$. If (3) holds then, taking into account that $g\left(e^{t X} A e^{-t X}\right)=e^{t X} g(A) e^{-t X}$, we obtain

$$
\left\|g(A)-e^{t X} g(A) e^{-t X}\right\|=\left\|g(A)-g\left(e^{t X} A e^{-t X}\right)\right\| \leq C\left\|A-e^{t X} A e^{-t X}\right\| .
$$

Dividing through by $t$ and taking the limit as $t \rightarrow 0$, we have that (4) holds.

Let $\|A\|=1, X \in B(H)$. For $r<1,\|[g(r A), X]\| \leq C\|[r A, X]\|$. Taking the limit as $r \rightarrow 1$, we obtain that (4) holds.

It follows from Proposition 1 that our aim is to construct a Fully Operator Lipschitz function with discontinuous derivative.

\section{Fully Operator Lipschitz Functions With Discontinuous Derivative}

Consider the following function on $D$ :

$$
h(1)=0 \quad \text { and } \quad h(z)=(z-1)^{2} \exp \left((z-1)^{-1}\right), \quad \text { for } z \in D, z \neq 1 .
$$

Since $\frac{x-1}{(x-1)^{2}+y^{2}}<0$, if $z=x+i y \in D \backslash 1$, we have that

$$
\begin{aligned}
\sup _{z \in D \backslash 1}\left|\exp \left((z-1)^{-1}\right)\right| & =\sup _{z \in D \backslash 1}\left|\exp \left(\frac{(x-1)-i y}{(x-1)^{2}+y^{2}}\right)\right| \\
& =\sup _{z \in D \backslash 1}\left|\exp \left(\frac{x-1}{(x-1)^{2}+y^{2}}\right)\right|\left|\exp \left(\frac{i y}{(x-1)^{2}+y^{2}}\right)\right| \\
& =\sup _{z \in D \backslash 1}\left|\exp \left(\frac{x-1}{(x-1)^{2}+y^{2}}\right)\right|<1 .
\end{aligned}
$$

The function $h$ is analytic on $D^{\circ}$ and continuous on $D$, since, by (5),

$$
|h(z)|=|h(x+i y)|=|z-1|^{2}\left|\exp \left((z-1)^{-1}\right)\right| \leq|z-1|^{2} \rightarrow 0,
$$


as $z \rightarrow 1$. Thus $h \in \mathcal{A}(D)$. We obtain similarly that

$$
\left|\frac{h(z)-h(1)}{z-1}\right|=|z-1|\left|\exp \left((z-1)^{-1}\right)\right| \leq|z-1| \rightarrow 0,
$$

as $z \rightarrow 1$, so $h^{\prime}(1)=0$. We also obtain that

$$
h^{\prime}(z)=2(z-1) \exp \left((z-1)^{-1}\right)-\exp \left((z-1)^{-1}\right), \quad \text { for } z \in D, z \neq 1 .
$$

We have, as above, that $(z-1) \exp \left((z-1)^{-1}\right) \rightarrow 0$, as $z \rightarrow 1$, while $\exp \left((z-1)^{-1}\right)$ does not have limit as $z \rightarrow 1$. Therefore $h^{\prime}$ is discontinuous at $z=1$.

Theorem 2. The function $h$ is Fully Operator Lipschitzian.

Proof. By Proposition 1, we only need to prove that (4) holds for $h$. For $0<\lambda<1$, set $h_{\lambda}(z)=h(\lambda z)$. Every $h_{\lambda}$ is analytic in a neighbourhood of $D$, so it belongs to $\mathcal{A}(D)$, and $\left\|h-h_{\lambda}\right\| \rightarrow 0$, as $\lambda \rightarrow 1$. Hence it follows from (2) that

$$
\begin{aligned}
\left\|[h(A), X]-\left[h_{\lambda}(A), X\right]\right\| & =\left\|\left[\left(h(A)-h_{\lambda}(A)\right), X\right]\right\| \\
& \leq 2\left\|h(A)-h_{\lambda}(A)\right\|\|X\| \leq 2\left\|h-h_{\lambda}\right\|\|A\|\|X\| \rightarrow 0 .
\end{aligned}
$$

For any $A \in \mathbf{B}_{1}$ and $X \in B(H)$,

$$
\begin{aligned}
\left\|\left[h_{\lambda}(A), X\right]\right\|= & \left\|\left[(\lambda A-\mathbf{1}) \exp \left((\lambda A-\mathbf{1})^{-1}\right)(\lambda A-\mathbf{1}), X\right]\right\| \\
= & \|[(\lambda A-\mathbf{1}), X] \exp \left((\lambda A-\mathbf{1})^{-1}\right)(\lambda A-\mathbf{1}) \\
& +(\lambda A-\mathbf{1})\left[\exp \left((\lambda A-\mathbf{1})^{-1}\right), X\right](\lambda A-\mathbf{1}) \\
& +(\lambda A-\mathbf{1}) \exp \left((\lambda A-\mathbf{1})^{-1}\right)[(\lambda A-\mathbf{1}), X] \| \\
\leq & 2 \lambda\|[A, X]\|\left\|\exp \left((\lambda A-\mathbf{1})^{-1}\right)\right\|\|\lambda A-\mathbf{1}\| \\
& +\left\|(\lambda A-\mathbf{1})\left[\exp \left((\lambda A-\mathbf{1})^{-1}\right), X\right](\lambda A-\mathbf{1})\right\| .
\end{aligned}
$$

We have that $\|\lambda A-\mathbf{1}\|<2$ and that the function $\exp \left((\lambda z-1)^{-1}\right)$ belongs to $\mathcal{A}(D)$. We obtain from (2) and (5) that

$$
\left\|\exp \left((\lambda A-\mathbf{1})^{-1}\right)\right\| \leq\left\|\exp \left((\lambda z-1)^{-1}\right)\right\| \leq \sup _{z \in D \backslash 1}\left|\exp \left((z-1)^{-1}\right)\right|<1 .
$$

Therefore

$$
\left\|\left[h_{\lambda}(A), X\right]\right\| \leq 4 \lambda\|[A, X]\|+\left\|(\lambda A-\mathbf{1})\left[\exp \left((\lambda A-\mathbf{1})^{-1}\right), X\right](\lambda A-\mathbf{1})\right\| .
$$

It follows from Lemma 2 of [5] that, for any $B \in B(H)$,

$$
[\exp (B), X]=\int_{0}^{1} \exp (t B)[B, X] \exp ((1-t) B) d t .
$$

If $B$ is invertible, then $B\left[B^{-1}, X\right] B=[X, B]$. Hence

$$
\begin{aligned}
& \left\|(\lambda A-\mathbf{1})\left[\exp \left((\lambda A-\mathbf{1})^{-1}\right), X\right](\lambda A-\mathbf{1})\right\| \\
& =\| \int_{0}^{1} \exp \left(t(\lambda A-\mathbf{1})^{-1}\right)(\lambda A-\mathbf{1})\left[(\lambda A-\mathbf{1})^{-1}, X\right](\lambda A-\mathbf{1}) \\
& \quad \exp \left((1-t)(\lambda A-\mathbf{1})^{-1}\right) d t \| \\
& \leq\|[X, \lambda A-\mathbf{1}]\| \int_{0}^{1}\left\|\exp \left(t(\lambda A-\mathbf{1})^{-1}\right)\right\|\left\|\exp \left((1-t)(\lambda A-\mathbf{1})^{-1}\right)\right\| d t .
\end{aligned}
$$


As in (7), we have that

$$
\left\|\exp \left(t(\lambda A-\mathbf{1})^{-1}\right)\right\|<1 \text { and }\left\|\exp \left((1-t)(\lambda A-\mathbf{1})^{-1}\right)\right\|<1 .
$$

Therefore

$$
\left\|(\lambda A-\mathbf{1})\left[\exp \left((\lambda A-\mathbf{1})^{-1}\right), X\right](\lambda A-\mathbf{1})\right\| \leq \lambda\|[A, X]\| .
$$

Hence we obtain from (8) that $\left\|\left[h_{\lambda}(A), X\right]\right\| \leq 5 \lambda\|[A, X]\|$. Combining this with (6), we conclude that $\|[h(A), X]\| \leq 5\|[A, X]\|$.

\section{REFERENCES}

1. D. R. Jocic, Integral representation formula for generalized normal derivation, Proc. Amer. Math. Soc. 127 (1999), 2303-2314. MR 99j:47026

2. B. E. Johnson and J. P. Williams, The range of a normal derivation, Pacific J. Math. 58 (1975), 105-122. MR 52:1390

3. E. Kissin and V. S. Shulman, Classes of Operator-smooth Functions. I. Operator Lipschitz Functions, preprint, (2000).

4. T. W. Palmer, Banach Algebras and the General Theory of ${ }^{*}$-algebras, vol. I, CUP, 1994. MR 95c:46002

5. R. Powers, A remark on the domain of an unbounded derivation of a $C^{*}$-algebra, J. Funct. Anal. 18 (1975), 85-95. MR 52:1334

6. B. Sz.-Nagy and C. Foias, Analyse Harmonique des Operateurs de l'espace de Hilbert, Academiai Kiado, Budapest, 1967. MR 37:778

7. J. P. Williams, Derivation ranges: open problems, Topics in Modern Operator Theory, (Timisoara/Herculane, 1980), 319-328, Operator Theory: Adv. Appl., 2, Birkhäuser, BaselBoston, MA, 1981. MR 83k:47027

School of Communications Technology and Mathematical Sciences, University of North London, Holloway, London N7 8DB, Great Britain

E-mail address: e.kissin@unl.ac.uk

School of Communications Technology and Mathematical Sciences, University of North London, Holloway, London N7 8DB, Great Britain - and - Department of Mathematics, Vologda State Technical University, Vologda, Russia

E-mail address: shulman_v@yahoo.com 